\title{
Editorial - Network modeling in psychopathology : hopes and challenges
}

(for the article Bortolon \& Raffard, Network analysis: are we moving towards a new conceptualization and treatment of mental disorders? 10.1016/i.encep.2019.06.001)

Yannick Morvan ${ }^{1,2}$, Eiko I. Fried ${ }^{3}$, Astrid Chevance ${ }^{4}$

1. Laboratoire Clinique Psychanalyse Développement (CLIPSYD), EA4430, UFR SPSE, Université Paris Nanterre, 92000 Nanterre, France

2. Institut de Psychiatrie et Neurosciences de Paris, INSERM UMR 1266, Laboratoire de Physiopathologie des Maladies Psychiatriques, Université de Paris,75014 Paris, France

3. Department of Clinical Psychology, Leiden University, Leiden, the Netherlands

4. University of Paris, CRESS, INSERM, INRA, F-75004 Paris, France

Clinical sciences do not only aim to describe psychological disorders, but also aim to explain them. And we are currently witnessing revisions of theoretical, methodological, and epistemological approaches. For instance, the ongoing debate about the use of psychiatric classifications, such as the DSM [1] and possible alternatives (Rdoc [2], HiTOP [3]), illustrates the epistemological tension between categorical and dimensional conceptualization of mental disorders. However, both approaches face a common problem: finding a way to reduce, but not ignore the complexity of mental illness to meet the challenge of making meaningful progress in research, treatment, and clinical decision making [4]. Numerous theoretical models have been proposed and explored since the beginning of psychiatry $[5,6]$, promising to increase our understanding of psychological disorders. A theory that has received a lot of attention in recent years is the network approach to psychopathology, arguing that mental illness is an emergent property that arises from causal interactions among symptoms (e.g. rumination>insomnia>fatigue>guilt) $[7,8]$. The last years have seen a growing number of statistical tools developed in the novel field of network psychometrics that have been used to study different aspects of psychopathology from the network perspective [9]. The network approach encompasses a) network theory, heavily inspired by long-standing theory in clinical psychology, and b) statistical 
models from complex dynamic systems theory that often have a long history in mathematics and physics, like the Ising Model $[10,11]$. Recent developments in computational science enable to test network theory by embracing the full scope of biopsychosocial complexity [12].

The network approach has gained momentum in part because it aligns with how clinicians think about mental illness-as causal networks of problems that influence each other-and promises to help clinicians understand the temporal dynamics of problems (e.g. symptoms) observed in clinical practice. While network models were originally applied mainly to cross-sectional data at the nomothetic (i.e. between-subjects) level, recent work has focused on studying within-person processes-including those of single individuals (i.e. idiographic) - and on formalizing clinical theories of mental disorders (e.g. panic disorder) and therapeutic tools (e.g. functional analysis) [13,14].

The paper of Bortolon \& Raffard, entitled Network analysis: are we moving towards a new conceptualization and treatment of mental disorders? [15], summarizes the advances in this emerging field of research for the francophone psychiatric community. This article is based on the work of Denny Borsboom, together with Angelique Cramer one of the main contributors to the field, who published several foundational pieces $[16,17]$.

In the first part, Bortolon \& Raffard present network analysis as a tool for exploring a third way of considering psychopathology between the medical model (i.e. symptoms are passive indicators of unobserved disease states, a reflective or essentialist approach) and the constructivist model (i.e. a disease is the construct formed by the combination of chosen characteristics such as symptoms, a formative or a constructivist approach) $[18,19]$. Statistical tools enable researchers to model psychopathology as a complex system of symptoms (based on the idea that these interaction may stem from causal processes), from which attractor states can emerge [9]. In network analysis, graphs are a useful way to represent relations (edges) between symptoms (nodes). Also, variables beyond symptoms can be modeled in networks, such as biological or environmental factors [20]. Network models have been used in many areas of clinical sciences, including depression [21], post-traumatic 
stress disorders (PTSD) [22], and psychosis [23]. The models have also been used to study comorbidity in psychiatric disorders [24]. For instance, in people with social anxiety disorders, work has highlighted the role of suicidal ideation, loss of interest, and loss of pleasure (depression symptoms) and avoidance of participating in small groups or going to a party, and fear of working (social anxiety symptoms) as potential bridges symptoms "through which activation issuing from one disorder potentially propagate to symptoms of the other disorder" [25].

The second part of the target article summarizes challenges for the network approach. Network analysis of psychopathology aims to escape the categorial notion of disorders, whereas diseases categories in medical classification are supposed to be distinct. However, most of the measurement tools used to measure symptoms were developed within a categorical framework in mind. The data they have produced are derived from this theory, and provide an obstacle for network analysis (and other dimensional approaches). Bortolon \& Raffard argue that network modeling is an important technique to identify transdiagnostic structures of psychopathology. They also encourage to study within-person processes using network approaches, for instance in randomized control trials where novel clinical targets are identified with centrality indices, and where potential changes of network structures over time are studied in detail [26]. Centrality indices in network models are statistical parameters that highlight which nodes might be most influential. For instance, if rumination emerges as a central node in depression for a given person, we might test whether intervening on rumination may also impact on other symptoms in case they are consequences of rumination. However, we would like to add that centrality indices have also been over-interpreted, and that we need to be careful in our inferences of statistical models. This has been summarized in recent work by Laura Bringmann and colleagues [27], and how well statistical indicators of centrality are correlated to clinical reality remains contentious [28]. Bringmann et al. made clear that in network analysis inferences should be drawn cautiously: "it is not enough to state that one wants to measure how central a node is, but one has to make explicit what is meant with being a central or important node, and what assumptions the centrality measure of choice entails" ([27], page 20). Further caution is required when it comes to 
causal inference. Even in temporal data, the fact that one event preceded another at best establishes Granger causality [8]. Unfortunately, inferences about causality have not always been drawn carefully in this emerging field, although this is likely a challenge in clinical sciences generally [29]. An important step would be that "empirical researchers gain a better understanding of network models and their assumptions" [30]. At an epistemological level, we do not believe that causality should be the only goal of clinical research. In fact, predicting the evolution of a system is possible without opening the black box of causality. For instance, the epistemology of complex systems (e.g. studied in cybernetics) paid little attention to the causal explanations of the system, with more focus put on how the system is behaving and evolving [31,32]. Another potential epistemological trap is the perpetual loop of codetermination between the definition of a clinical entity and its measurement, a problem that of course goes beyond network analysis. Other challenges should be mentioned, such as confirmation bias and exploiting ambiguity in what is acceptable practice by engaging in questionable research practices, which may be mitigated via pre-registrations [33,34].

Finally, Bortolon \& Raffard highlight the question of stability and replicability of psychopathological network as an important aspect of clinical science. We agree that discovering robust, replicable phenomena in clinical sciences is a crucial goal, which includes phenomena identified via network analysis. Pre-registration, open data and code can help achieve this goal to some degree, and enhances transparency and reproducibility [35]. Overall, the emerging network literature has been doing comparably well in sharing data, code, and materials; for instance, when 22 authors of network papers were recently approached for re-analysis of their data, 18 made the data available without any hesitation [36]. Therefore, network analysis has not only become a growing clinical field, but also a welcoming community of like-minded researchers, with the Facebook group Psychological Dynamics (Facebook.com/groups/psychologicaldynamics) being a great example. For further reading, Eiko Fried's blog Psych-Networks.com, the website Psychosystems.org by the lab of Denny Borsboom, and numerous other sites (e.g. by Claudia van Borkulo, Payton Jones, Donald Williams, Sacha Epskamp and others) are good examples of such open practices [37]. 
As a conclusion, using network analysis to model psychopathology as complex systems of interacting biopsychosocial problems may be one important way forward-as long as we remain cautious about inferences we draw from data.

\section{References}

[1] Kendell R, Jablensky A. Distinguishing between the validity and utility of psychiatric diagnoses. Am J Psychiatry 2003;160:4-12.

[2] Insel T, Cuthbert B, Garvey M, Heinssen R, Pine DS, Quinn K, et al. Research Domain Criteria (RDoC): Toward a New Classification Framework for Research on Mental Disorders. Am J Psychiatry 2010;167:748-51. doi:10.1176/appi.ajp.2010.09091379.

[3] Kotov R, Waszczuk MA, Krueger RF, Forbes MK, Watson D, Clark LA, et al. The hierarchical taxonomy of psychopathology (HiTOP): A dimensional alternative to traditional nosologies. J Abnorm Psychol 2017;126:454-77. doi:10.1037/abn0000258.

[4] Clark LA, Cuthbert B, Lewis-Fernández R, Narrow WE, Reed GM. Three Approaches to Understanding and Classifying Mental Disorder: ICD-11, DSM-5, and the National Institute of Mental Health's Research Domain Criteria (RDoC). Psychol Sci Public Interes 2017;18:72-145. doi:10.1177/1529100617727266.

[5] Berrios GE. Classifications in psychiatry: a conceptual history. Aust N Z J Psychiatry 1999;33:145-60.

[6] Kendler KS. An historical framework for psychiatric nosology. Psychol Med 2009;39:1935-41. doi:10.1017/\$0033291709005753.

[7] Borsboom D, Cramer AOJ. Network Analysis: An Integrative Approach to the Structure of Psychopathology. Annu Rev Clin Psychol 2013;9:91-121. doi:10.1146/annurev-clinpsy050212-185608. 
[8] Epskamp S, van Borkulo CD, van der Veen DC, Servaas MN, Isvoranu AM, Riese H, et al. Personalized Network Modeling in Psychopathology: The Importance of Contemporaneous and Temporal Connections. Clin Psychol Sci 2018;6:416-27. doi:10.1177/2167702617744325.

[9] Fried E, van Borkulo CD, Cramer AOJ, Boschloo L, Schoevers RA, Borsboom D. Mental disorders as networks of problems: a review of recent insights. Soc Psychiatry Psychiatr Epidemiol 2017;52. doi:10.1007/s00127-016-1319-z.

[10] Van Borkulo CD, Borsboom D, Epskamp S, Blanken TF, Boschloo L, Schoevers RA, et al. A new method for constructing networks from binary data. Sci Rep 2014;4:1-10. doi:10.1038/srep05918.

[11] Borsboom D. The meaning of model equivalence: Network models, latent variables, and the theoretical space in between. Psych Networks Blog 2017. https://psychnetworks.com/meaning-model-equivalence-network-models-latent-variables-theoreticalspace/ (accessed July 13, 2019).

[12] Engel GL. The need for a new medical model: a challenge for biomedicine. Science (80- ) 1977;196:129 LP - 136. doi:10.1126/science.847460.

[13] Burger J, Van Der Veen DC, Robinaugh DJ, Quax R, Riese H, Schoevers RA, et al. Bridging the Gap Between Complexity Science and Clinical Practice by Formalizing Idiographic Theories: A Computational Model of Functional Analysis. 2019. doi:10.31234/osf.io/gw2uc.

[14] Robinaugh DJ, Haslbeck JMB, Waldorp LJ, Kossakowski JJ, Fried El, Millner AJ, et al. Advancing the Network Theory of Mental Disorders: A Computational Model of Panic Disorder. 2019. doi:10.31234/osf.io/km37w.

[15] Bortolon C, Raffard S. Network analysis: are we moving towards a new conceptualization and treatment of mental disorders? Encephale 2019.

[16] Cramer AOJ, Waldorp L, van der Maas HLJ, Borsboom D. Comorbidity: A network perspective. 
Behav Brain Sci 2010;33:137-50. doi:10.1017/S0140525X09991567.

[17] Borsboom D. A network theory of mental disorders. World Psychiatry 2017;16:5-13. doi:10.1002/wps.20375.

[18] Fried E. What are psychological constructs? On the nature and statistical modeling of emotions, intelligence, personality traits and mental disorders 2017. doi:10.17605/OSF.IO/PP6XK.

[19] Kendler KS. DSM disorders and their criteria: how should they inter-relate? Psychol Med 2017;47:2054-60. doi:10.1017/\$0033291717000678.

[20] Fried El, von Stockert S, Haslbeck JMB, Lamers F, Schoevers RA, Penninx BWJH. Using network analysis to examine links between individual depressive symptoms, inflammatory markers, and covariates. Psychol Med 2019:1-9. doi:10.1017/S0033291719002770.

[21] Fried El, Epskamp S, Nesse RM, Tuerlinckx F, Borsboom D. What are "good" depression symptoms? Comparing the centrality of DSM and non-DSM symptoms of depression in a network analysis. J Affect Disord 2016;189:314-20. doi:10.1016/j.jad.2015.09.005.

[22] Fried El, Eidhof MB, Palic S, Costantini G, Huisman-van Dijk HM, Bockting CLH, et al. Replicability and Generalizability of Posttraumatic Stress Disorder (PTSD) Networks: A CrossCultural Multisite Study of PTSD Symptoms in Four Trauma Patient Samples. Clin Psychol Sci 2018;6:335-51. doi:10.1177/2167702617745092.

[23] Isvoranu A-M, Guloksuz S, Epskamp S, van Os J, Borsboom D. Toward incorporating genetic risk scores into symptom networks of psychosis. Psychol Med 2019. doi:10.1017/S003329171900045X.

[24] Jones PJ, Ma R, McNally RJ. Bridge Centrality: A Network Approach to Understanding Comorbidity. Multivariate Behav Res 2019;0:1-15. doi:10.1080/00273171.2019.1614898. 
[25] Heeren A, Jones PJ, McNally RJ. Mapping network connectivity among symptoms of social anxiety and comorbid depression in people with social anxiety disorder. J Affect Disord 2018;228:75-82. doi:10.1016/j.jad.2017.12.003.

[26] Epskamp S, Waldorp LJ, Mõttus R, Borsboom D. The Gaussian Graphical Model in CrossSectional and Time-Series Data. Multivariate Behav Res 2018;53:453-80. doi:10.1080/00273171.2018.1454823.

[27] Bringmann L, Elmer T, Epskamp S, Krause R, Schoch D, Wichers M, et al. What do centrality measures measure in psychological networks? 2018. doi:10.13140/RG.2.2.25024.58884.

[28] Rodebaugh TL, Tonge NA, Piccirillo ML, Fried E, Horenstein A, Morrison AS, et al. Does centrality in a cross-sectional network suggest intervention targets for social anxiety disorder? J Consult Clin Psychol 2018;86:831-44. doi:10.1037/ccp0000336.

[29] Ryan O, Bringmann LF, Schuurman NK. The Challenge of Generating Causal Hypotheses Using Network Models. 2019. doi:10.31234/osf.io/ryg69.

[30] Fried El, Cramer AOJ. Moving Forward: Challenges and Directions for Psychopathological Network Theory and Methodology. Perspect Psychol Sci 2017;12:999-1020. doi:10.1177/1745691617705892.

[31] Wiener N. Cybernetics or Control and Communication in the Animal and the Machine. Technology Press; 1948.

[32] Yarkoni T, Westfall J. Choosing Prediction Over Explanation in Psychology: Lessons From Machine Learning. Perspect Psychol Sci 2017;12:1100-22. doi:10.1177/1745691617693393.

[33] Gelman A, Loken E. The garden of forking paths: Why multiple comparisons can be a problem, even when there is no "fishing expedition" or "p-hacking" and the research hypothesis was posited ahead of time. 2013. 
[34] Tackett JL, Brandes CM, King KM, Markon KE. Psychology's Replication Crisis and Clinical Psychological Science. Annu Rev Clin Psychol 2019;15. doi:10.1146/annurev-clinpsy-050718095710.

[35] Borsboom D, Fried El, Epskamp S, Waldorp L, van Borkulo CD, van der Maas HLJ, et al. False alarm? A comprehensive reanalysis of "evidence that psychopathology symptom networks have limited replicability" by Forbes, Wright, Markon, and Krueger (2017). J Abnorm Psychol 2017;126:989-99. doi:10.1037/abn0000306.

[36] Haslbeck JMB, Fried El. How predictable are symptoms in psychopathological networks? A reanalysis of 18 published datasets. Psychol Med 2017;47:2767-76. doi:10.1017/\$0033291717001258.

[37] Tackett JL, Brandes CM, Reardon KW. Leveraging the Open Science Framework in clinical psychological assessment research. Psychol Assess 2019;3:1-4. doi:10.1037/pas0000583. 